\title{
A COCKTAIL OF MCM2 AND TOP2A, P16INK4a AND KI-67 AS BIOMARKERS FOR THE IMPROVED DIAGNOSIS OF CERVICAL INTRAEPITHELIAL LESION
}

\author{
Qi-chang Yang, Yan Zhu, Hong-Bing Liou, XiaO-juan Zhang, Yi Shen, XiaO-hua Ji
}

Department of Pathology, The $2^{\text {nd }}$ Affiliated Hospital of Nantong University, Nantong, China

\begin{abstract}
The purpose of this paper was to explore the immunohistochemistry (IHC) results for a cocktail of minichromosome maintenance protein 2 (MCM2) and topoisomerase II $\alpha$ (TOP2A), p16INK4a and Ki-67 as biomarkers for the diagnosis of cervical intraepithelial neoplasia (CIN), improving the routine interpretation of cervical histopathology. 133 cases of CIN were collected from the archival data. All routine hematoxylin and eosin (HE)-stained slides of the subjects were re-examined independently by three senior pathologists, to provide a "consensus diagnosis". Immunohistochemistry for the three biomarkers was performed, and the results were reviewed independently of the corresponding archival diagnosis to make a "diagnosis assisted by IHC" by the original pathological practitioners. The diagnosis accordance rate of the archival original diagnosis with the "consensus diagnosis" and the "diagnosis assisted by IHC" with the "consensus diagnosis" were verified by Fisher's exact test. The results showed that raw agreement between the original HE diagnosis and the "consensus diagnosis" was $88.55 \%$, and raw agreement between the "diagnosis assisted by IHC" and the "consensus diagnosis" was 95.78\%. The latter was significantly higher than the former (Fisher's exact test, $p=0.023$ ). In conclusion, the three biomarkers had a high degree of sensitivity and specificity, and appear to be a useful and reliable diagnostic adjunct to improve the routine diagnosis, and reduce inter-observer variability in cervical biopsy specimens.
\end{abstract}

Key words: cervical intraepithelial neoplasia, antibody cocktail, p16INK4a, Ki-67, immunostaining, diagnostic accuracy.

\section{Introduction}

Cervical cancer is a common cancer in women caused by high-risk human papilloma virus ( $\mathrm{Hr}-\mathrm{HPV})$. Sustaining infection of $\mathrm{Hr}-\mathrm{HPV}$ leads to precancerous lesions, cervical intraepithelial neoplasia (CIN) or cervical cancer $[1,2]$. Cervical intraepithelial neoplasia is a continuous process from CIN to cervical cancer $[1,3]$ and it is divided into normal, CIN 1, CIN 2, CIN 3 (including carcinoma in situ) and cervical cancer $[1,3]$. Therefore, accurate diagnosis of CIN and treatment of precancerous lesions is critical to the discovery and prevention of cervical cancer. The CIN nomenclature is primarily based on a subjective estimation of the thickness, from the base to the surface of the epithelium, of the percentage substitution of differentiating epithelial cells by proliferating epithelial cells: lower than $1 / 3$ refers to CIN 1 , between $1 / 3$ and $2 / 3$ refers to CIN 2 , and over 2/3 means CIN 3. Cervical intraepithelial neoplasia 1 is the histopathological indication of HPV infection by carcinogenic and non-carcinogenic HPV, while CIN 2 and CIN 3 are considered as precancerous diagnoses [1, 3]. Although CIN 2 is a heterogeneous lesion, containing both precancerous and transient lesions, it is still treated as the critical criterion to prevent cancer [3]. Therefore, the results of the histopathological diagnoses of cervical epithelium lesion guide the subsequent clinical management and early treatment of CIN. 
The histopathological interpretation of CIN is subject to a high level of inter-observer variability. It is often mixed up with reactive/reparative epithelial changes, immature squamous metaplasia, and atrophy $[1,4,5]$. To increase the accuracy of CIN diagnosis, it is especially necessary and significant to use biomarkers as an objective indicator to help in histopathological diagnoses of CIN [3, 6-11].

P16INK4a (p16) is a kind of anti-oncogene and considered as a preferable substitute marker for $\mathrm{Hr}-\mathrm{HPV}$ infection [1-10, 12-18]. As a cyclin-dependent kinase inhibitor, p16 competes with cyclin D1 to combine with CDK $4 / 6$ and specifically suppresses the activity of CDK4, inhibiting the CDK-induced phosphorylation of $\mathrm{pRb}$ and arresting the cell transition from G1 phase to $S$ phase, thereby playing a role in feedback control of mitosis. Also, p16 is included in the process of cervical lesions caused by HPV infection. When cervical cancer and CIN occur, the HPV E7 proteins cause dysfunction of $\mathrm{pRb}$, causing over-expression of $\mathrm{p} 16$ protein yet being unable to control the cell cycle. P16 shows a high rate of positive results in high grade CIN while it shows a low rate of positive results and weak expressions in low grade CIN [9, 13, 19].

Recently, a biomarker cocktail containing antibodies against minichromosome maintenance protein 2 (MCM2) and topoisomerase II (TOP2A) has been reported as a potential diagnostic adjunct for CIN $[4,12,13,20]$. During DNA replication, MCM2 functions by loading the complex onto DNA before replication and unwinding the DNA through helicase activity to permit DNA synthesis. Topoisomerase II $\alpha$ is responsible for the enzymatic unlinking of DNA strands during replication. Topoisomerase II $\alpha$ and MCM2 work together in the regulation of DNA replication during S-phase and are overexpressed when S-phase cell cycle induction is aberrant. During the transcriptional activation of the aberrant cell cycle, levels of MCM2 and TOP2A increase in proliferating cells. They have been shown to be overexpressed in various dysplastic and malignant processes, including cervical neoplasia related to high-risk HPV. Any single application of MCM2 or TOP2A will leave out some squamous epithelial lesions, so it is generally approved to apply the cocktail of MCM2 and TOP2A (ProExC) by immunostaining in CIN diagnosis $[13,20]$.

In the present study, the cocktail of MCM2 and TOP2A, p16 and $\mathrm{Ki}-67$, an antigen expressed in the nuclei of proliferating cells and detected with MIB-1, were used as diagnostic adjuncts in cervical biopsy specimens. The clinical values of these biomarkers' expression were evaluated in order to explore whether they may provide objective standards to reduce diagnostic variability of cervical biopsy evaluations, and improve the routine interpretation of cervical histopathology.

\section{Material and methods}

\section{Data collection and review}

One hundred and twenty-seven cases of CIN were selected from the archive of our hospital between July 2006 and July 2008 as subjects. Cervical biopsy, cone and curettage specimens were collected. Patients' average age was 44 years (range 20-86 years). Most of the subjects did not receive any immunohistochemical staining at the time of histological diagnosis. All routine hematoxylin and eosin (HE)-stained slides of the subjects were reviewed under a multi-viewing microscope and discussed by three experienced pathologists blinded to any clinical information except for patient age. A "consensus diagnosis" was fulfilled when at least two of the reviewing pathologists achieved agreement on the result. If the three pathologists all had different opinions, the result was confirmed by immunohistochemical expression. We had the following "consensus diagnoses": 29 cases of CIN 1, 35 cases of CIN 2, and 63 cases of CIN 3. Nineteen cases of chronic cervicitis (four cases with squamous metaplasia and five cases with immature squamous metaplasia included) and 20 cases of invasive squamous cell carcinoma (SCC) during the same time span were included as the control group.

\section{Immunohistochemistry}

The specimens were fixed in $10 \%$ neutral-buffered formalin, processed using standard procedures and embedded in paraffin, and $4-\mu \mathrm{m}$-thick serial sections were cut. Tissue sections were deparaffinized and rehydrated through grade alcohols. Antigen retrieval was carried out with $1 \mathrm{mM}$ EDTA ( $\mathrm{pH}$ 8.0) and pressure cooker heat induction $\left(122-125^{\circ} \mathrm{C}\right)$ for $30 \mathrm{~s}$ at $15-24$ psi. Application of the primary antibody was followed by incubation with the UltraSensitive ${ }^{\mathrm{TM}}$ S-P (Mouse/Rabbit) detection system with $\mathrm{DAB}$ as the chromogen and haematoxylin counterstaining. Rabbit anti-human MCM2 monoclonal antibody (clone SP50, from Spring Bioscience Corporation, dilution of $1: 100$ ) and mouse anti-human TOP2A monoclonal antibody (clone 3F6, from Spring Bioscience Corporation, dilution of $1: 30$ ) were mixed into a cocktail antibody after dilution [13]. Mouse anti-human p16INK4a monoclonal antibody (clone 16P04/JC2), with working dilution of $1: 200$, and $\mathrm{Ki}-67$, identified using the mouse monoclonal antibody MIB-1, were products from Zymed Laboratories, Inc. We substituted PBS for primary antibodies as a negative control and used known cervical squamous cell carcinoma sections as a positive control. All the negative control and positive control sections were included in each staining run for all the three antibodies.

\section{Immunohistochemical scoring}

For the antibody cocktail of MCM2 and TOP2A, and $\mathrm{Ki}-67$, positive expression was located in the nucleus of 
lesion cells. Positive expression of p16 was located in both the nucleus and the cytoplasm. The immunohistochemical (IHC) results for all antibodies were judged referring to Pinto \& Shi's experiment $[4,13]$, with slight adaptation. Semi-quantitative report was used to analyze the results of staining by intensity and distribution. The staining intensity was graded as weak (light brown) or strong (dark brown). The distribution was graded according to the location of positive cells horizontally and vertically on epithelium, staining on basal 1-2 layers of cells labelled as $1+$, staining of lower one-third of cells as $2+$, staining of lower two-thirds of cells as $3+$ and more than the lower two-thirds to full thickness of the epithelial lesion as $4+$. The following situations were interpreted to be negative: positive cells were less than 5\%; the antibody cocktail and $\mathrm{Ki}-67$ staining was distributed on basal 1-2 layers of cells; p16 was stained only on cytoplasm. According to David's method [12], negative results of the antibody cocktail, p16 and $\mathrm{Ki}-67$ were considered as without CIN; the antibody cocktail $(2+), \mathrm{p} 16(1+\& 2+)$ and $\mathrm{Ki}-67(2+)$ were all CIN 1 ; the antibody cocktail, p16, and $\mathrm{Ki}-67$ ( $\geq 3+$ ) were $\mathrm{CIN} 2-3$, in which immunostaining scoring $4+$ was judged as CIN 3 .

The pathologists who made the original diagnosis by HE-stained slides were designated to make a "diagnosis assisted by IHC" combining the HE-stained section and IHC but without the previous histological diagnosis.

\section{Statistical analysis}

Stata 7.0 was applied for data analysis. $\mathrm{P}<0.05$ was considered statistically significant. Univariate $\chi^{2}$ tests and Spearman analysis were used to assess the correlations between CIN and immunohistochemical expression of three biomarkers. The differences between the diagnosis accordance rate of the archival original diagnosis, "diagnosis assisted by IHC" and "consensus diagnosis" were verified by Fisher's exact test.

\section{Results}

Table I shows the expression of the antibody cocktail, p16INK4A and Ki-67 on cervical lesions. For the antibody cocktail, and $\mathrm{Ki}-67$, positive expression was located in the nucleus of basal 1-2 layers of cells and lesion cells without cytoplasm stained (Figs. 1-3). Positive expression of p16 was located in both the nucleus and the cytoplasm, partial or whole epithelial tissue stained (Figs. 1-3). In the negative control group, five cases of immature squamous metaplasia were negative for the cocktail antibody, only expressed on basal 1-2 layers of cells. For p16, these cases were negative except one case with small clusters of cells with halo colouring, while 3 cases were negative and 2 cases were $2+$ for $\mathrm{Ki}-67$.

The differences between the expressions of the antibody cocktail, p16 and Ki-67 in N-CIN, CIN 1, CIN 2, CIN 3 and squamous cell carcinoma respectively had statistical significance $\left(\chi^{2}=271.76,290.56,143.84\right.$, $\mathrm{p}=0.000)$. Spearman's rank correlation coefficient showed that the degree of expression positively correlated with cervical lesion (respectively $\mathrm{r}=0.78,0.79$, $0.75, \mathrm{p}=0.000)$.

Table II shows the comparison of consistency between original HE diagnosis and the "consensus diagnosis", and consistency between "diagnosis assisted by IHC" and "consensus diagnosis". Raw agreement of the former was $88.55 \%$ (147/166) (95\% confident interval of $82.70-92.97 \%$ ) while raw agreement of the latter was $95.78 \%$ (159/166) (95\% confident interval of 91.51-98.29\%). The latter was significantly higher than the former (Fisher's exact test, $\mathrm{p}=0.023$ ).

In some cases the diagnosis was corrected based on IHC and the "consensus diagnosis":

Normal group: One case initially diagnosed as normal by the primary pathologist was corrected to CIN 1 based on IHC, in which ProExC, p16 and Ki-67 all showed $2+$.

CIN 1 group: The consensus diagnosed 29 cases. Of these 29 cases the primary pathologists had initially diagnosed 27 as CIN 1; one case was diagnosed as normal and one as CIN 2. After IHC the one called CIN 2 remained the same and the one called normal was corrected, resulting in 28 cases correctly diagnosed as CIN 1. Improvement in diagnosis: one case.

CIN 2 group: The consensus diagnosed 35 cases. Of these 35 cases the primary pathologist had initially di-

Table I. Expressions of antibody cocktail, p16 and Ki-67 for different cervical lesions

\begin{tabular}{|c|c|c|c|c|c|c|c|c|c|c|c|c|c|}
\hline \multirow{2}{*}{$\begin{array}{l}\text { DIAG } \\
\text { NOSIS }\end{array}$} & \multirow[b]{2}{*}{$\mathbf{N}$} & \multicolumn{4}{|c|}{ ANTIBODY COCKTAIL } & \multicolumn{4}{|c|}{ P16 } & \multicolumn{4}{|c|}{ KI-67 } \\
\hline & & Negative & $2+$ & $3+$ & $4+$ & Negative & $1 \& 2+$ & $3+$ & $4+$ & Negative & $2+$ & $3+$ & $4+$ \\
\hline $\mathrm{N}-\mathrm{CIN}$ & 19 & 19 & 0 & 0 & 0 & 18 & 1 & 0 & 0 & 14 & 3 & 2 & 0 \\
\hline CIN 1 & 29 & 2 & 23 & 4 & 0 & 2 & 27 & 0 & 0 & 15 & 10 & 4 & 0 \\
\hline CIN 2 & 35 & 0 & 4 & 17 & 14 & 0 & 3 & 13 & 19 & 0 & 3 & 10 & 22 \\
\hline CIN 3 & 63 & 0 & 0 & 25 & 38 & 0 & 0 & 22 & 41 & 0 & 0 & 11 & 52 \\
\hline SCC & 20 & 0 & 0 & 2 & 18 & 0 & 0 & 0 & 20 & 0 & 0 & 3 & 17 \\
\hline Total & 166 & 21 & 27 & 48 & 70 & 20 & 31 & 35 & 80 & 29 & 16 & 30 & 91 \\
\hline
\end{tabular}



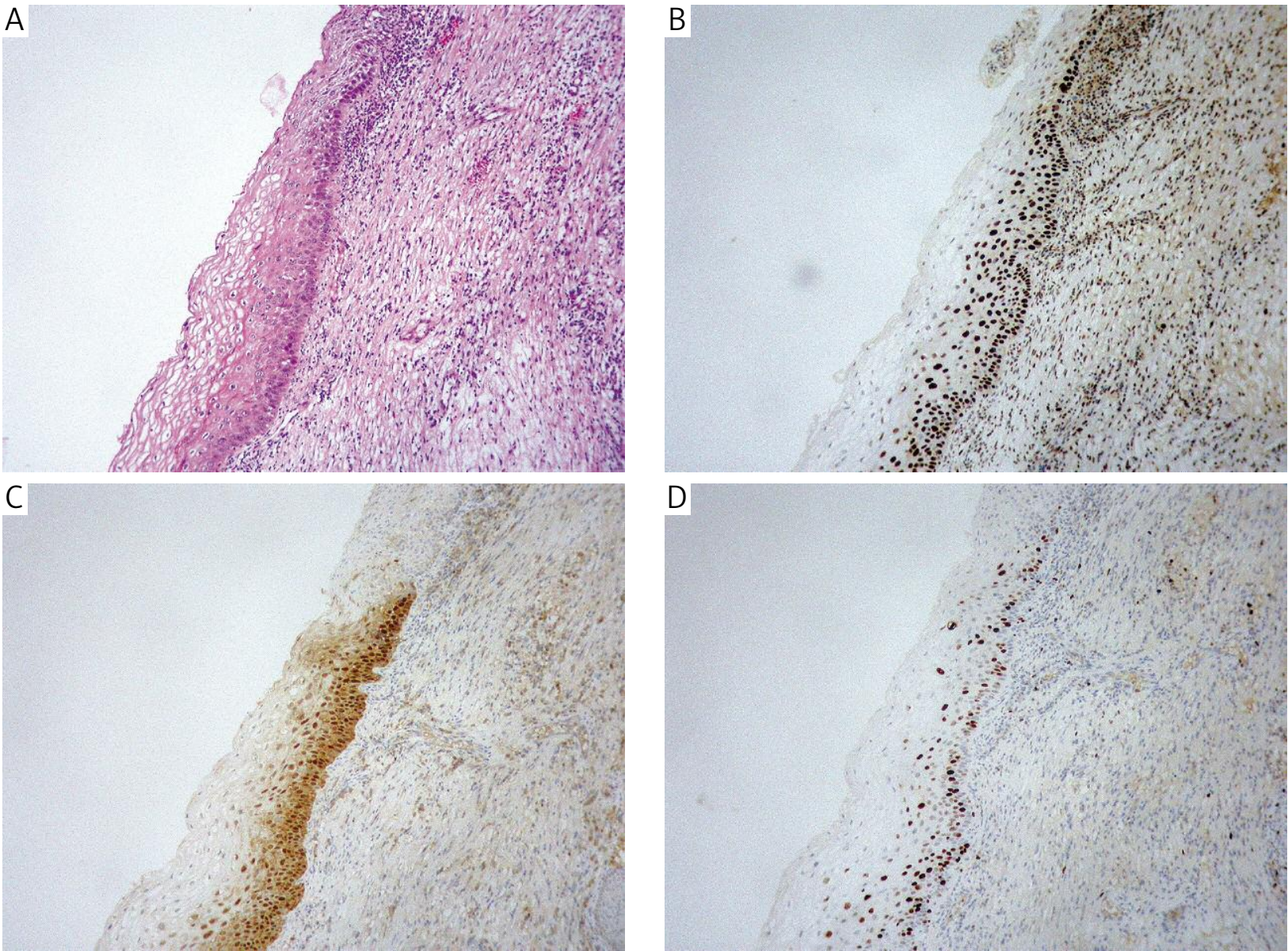

Fig. 1. CIN 1: A - HE, 1/3 cells of cervical squamous epithelium show slight atypia, N/C ratio increased, while cervical epithelium in the upper part of sight normal; B - ProExC, positive expression seen on fundus and its vicinity cells and nucleus of atypical epithelium cells and nearly $1 / 2$ of cells positive $(2+)$, only fundus and its vicinity cells stained in normal epithelium; $\mathrm{C}-\mathrm{p} 16$, lower $1 / 3$ of cells in epithelium positive and positive expression seen on karyon and cytoplasm with continuous stain; D - Ki-67, lower $1 / 3$ of cells positive, and expression seen on fundus cells and nucleus of atypical epithelium cells with sparse stain, few had 1/2 cells stained. Magnification $200 \times$

agnosed 26 as CIN 2, 7 as CIN 1 and 2 as CIN 3. After IHC 32 cases were correctly diagnosed as CIN 2 and 3 cases were under-diagnosed as CIN 1. Improvement in diagnosis: 6 cases.

CIN 3 group: The consensus diagnosed 63 cases. Of these 63 cases the primary pathologist initially diagnosed correctly 55 cases as CIN 3 and 8 cases were under-diagnosed as CIN 2. After IHC 60 cases were correctly diagnosed as CIN 3. Improvement in diagnosis: 5 cases.

It was found that IHC for the cocktail antibody, p16 and $\mathrm{Ki}-67$ had a high degree of sensitivity and specificity for interpretation of cervical histopathology, and the diagnosis assisted by IHC had a higher consistency rate than the original HE diagnosis.

\section{Discussion}

The histological diagnosis of CIN is inevitably affected by subjective factors of the pathologist $[3,6]$. It is nec- essary to distinguish normal from CIN of any grade, and benign or lower-grade CIN, which is mostly transient dysplasia (CIN 1), from high-grade CIN. P16 is a preferable substitute indicator for $\mathrm{Hr}-\mathrm{HPV}$ infection, and its expression in CIN is widely researched and reported [1-10, 12-18]. According to previous studies [3], p16 showing diffuse strong positivity was highly sensitive to CIN 3 and CIN 2 but insensitive to CIN 1. Branea $[15]$ believed that p16 could be considered as a specific indicator for high-grade $\mathrm{CIN}$, its positive predictive value being $100 \%$. Therefore, $\mathrm{p} 16$ is useful in distinguishing high-grade CIN from low-grade CIN but probably not useful in distinguishing CIN 1 from nonCIN. In comparison, $\mathrm{Ki}-67$ is also sensitive for CIN 3 and CIN 2, but has less specificity. Additionally, combining p16 and Ki-67 diagnoses does not show better results than using p16 alone. Ma et al. [21] also discovered that as the CIN evolves to a higher grade, p16 protein expression became stronger, manifesting a linearly relation, which indicates a certain diagnostic value of $\mathrm{p} 16$ 

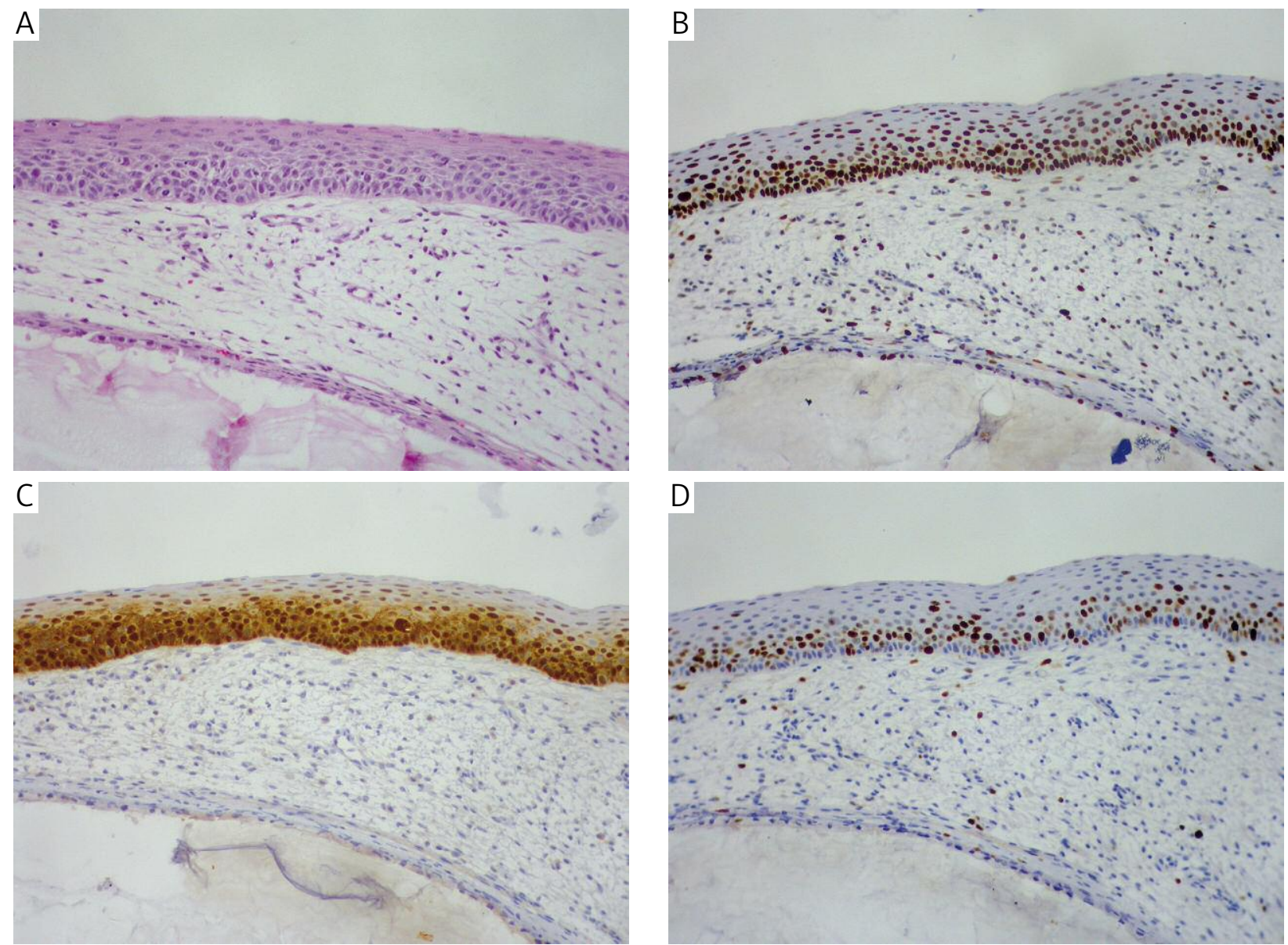

Fig. 2. CIN 2: A - HE, 1/2 of cells of cervical squamous epithelium show atypia, N/C ratio increased; $\mathbf{B}-\mathrm{ProExC}$, positive expression seen on nucleus of atypical epithelium cells and $1 / 2$ to $2 / 3$ of cells positive $(3+)$; $\mathbf{C}-\mathrm{P} 16$, lower $1 / 2$ of cells in atypical epithelium positive and expression seen on karyon and cytoplasm with continuous and moderate stain $(2+)$; D $-\mathrm{Ki}-67$, more than $1 / 2$ of cells in atypical epithelium positive $(3+)$. Magnification $200 \times$

IHC for CIN. However, O’Neill et al. \{22\} held that applying p16 alone is neither $100 \%$ specific nor sensitive for a given lesion. And only p16 positivity combined with other indicators can confirm the diagnosis.

Recently multiple reports $[4,12,13,20]$ have shown that an antibody cocktail of MCM2 and TOP2A (ProExC) is a reliable indicator for high-grade CIN. It is highly sensitive and specific to high-grade CIN and may help to predict lesions which tend to progress. A report [13] compared the expression of ProExC and of p16 in 62 cervical biopsy specimens. It found that p16 is more sensitive and specific for identification of highgrade squamous intraepithelial lesions while ProExC is more sensitive to low-grade squamous intraepithelial lesions, which makes combing ProExC and p16 have the highest diagnostic value for both high-grade and low-grade squamous intraepithelial lesions. According to Pinto's study [4], ProExC has the same high sensitivity and specificity as p16 to high-grade CIN. Its false positive rate is markedly lower than $\mathrm{Ki}-67$ for $\mathrm{N}$-CIN, so it is more effective than $\mathrm{Ki}-67$ in distinguishing reactive epithelial changes from squamous intraepithe- lial lesions. Also, Kelly’s study [20] proposed that lowgrade squamous intraepithelial lesions could be separated into two groups based on their expression of ProExC, of which the positive group was more similar to high-grade lesions in its biological behaviour. Because of the low false positive rate, the antibody cocktail has higher positive predictive value than $\mathrm{Hr}-\mathrm{HPV}$ detection in CIN diagnosis.

Compared with p16, Ki-67 used to detect cervical epithelial dysplasia is sensitive but less specific, which makes it unable to distinguish low-grade CIN and highgrade CIN, so it cannot be applied alone in identifying cervical epithelial dysplasia $[13,23,24]$. It was reported that Ki-67 quantitative assessment may provide information on the progression of CIN 1 and CIN 2 $[4,10,19]$.

In the present study, differences among the expressions of the antibody cocktail, p16 and Ki-67 in N-CIN, CIN 1, CIN 2, CIN 3 and squamous carcinoma groups had statistical significance and were positively related to the grading of CIN. They were the same results as the references, evincing values of IHC 

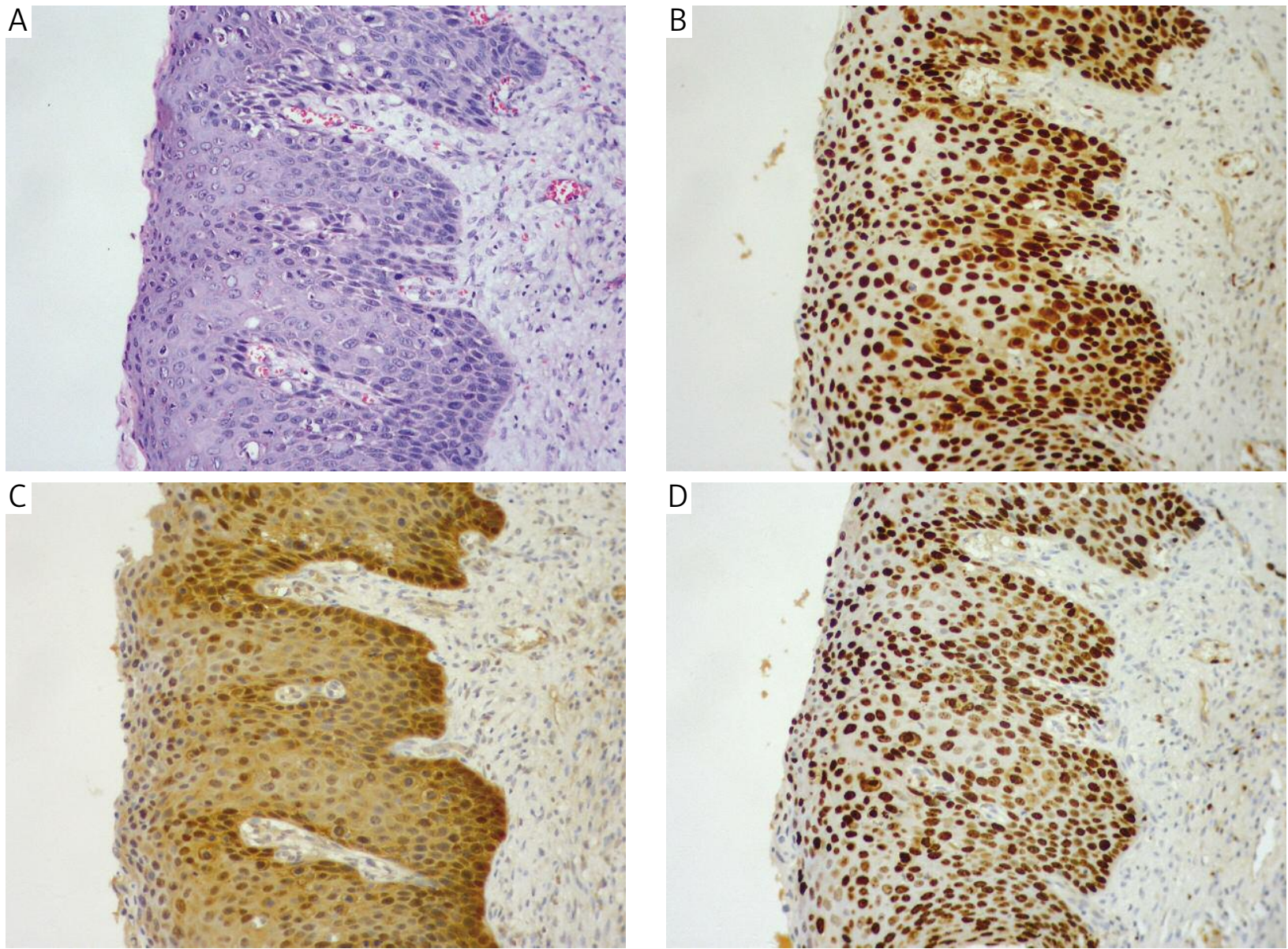

Fig. 3. CIN 3/carcinoma in situ: A - HE, whole layer of cells on cervical squamous epithelium show atypia and nucleus deeply stained; B - ProExC, strong positive expression on whole layer of cells on cervical squamous epithelium $(4+)$; C - P16, strong positive (4+); D - Ki-67, strong positive (4+). Magnification 200X

indicators such as the antibody cocktail, p16 and Ki67 in diagnosing cervical intraepithelial lesions.

Subjectivity of pathologists and diagnostic variability are inevitable. However, in clinical practice, the necessity of using IHC indicators to assist in histopathological diagnoses, as well as how much they can reduce the diagnosis deviation and improve the diagnostic repeatability, has been discussed less in recent literature. In fact, part of the decision of whether to use special immunohistochemical staining in a given case is completely dependent on whether the pathologist thinks there is a diagnostic problem. [3] It was reported that although many members of the faculty had worked together continuously for a long time to have an accordant diagnosis, the overall impression of diagnostic variation is still obvious in histological diagnosis. The relative tendency was CIN 2 being underdiagnosed. In Bergeron's study [6], 500 cases of cervical biopsy specimens in HE-stained slides were independent diagnoses by twelve pathologists, which were

Table II. Comparison between original diagnosis, diagnosis assisted by IHC and the "consensus diagnosis"

\begin{tabular}{|c|c|c|c|c|c|c|c|c|c|c|c|}
\hline \multicolumn{2}{|c|}{ CONSENSUS } & \multicolumn{5}{|c|}{ ORIGINAL DIAGNOSIS } & \multicolumn{5}{|c|}{ DIAGNOSIS ASSISTED BY IHC } \\
\hline DIAGNOSIS & $\mathrm{N}$ & N-CIN & CIN 1 & CIN 2 & CIN 3 & SCC & $\mathrm{N}-\mathrm{CIN}$ & CIN 1 & CIN 2 & CIN 3 & SCC \\
\hline N-CIN & 19 & 19 & 0 & 0 & 0 & 0 & 19 & 0 & 0 & 0 & 0 \\
\hline CIN 1 & 29 & 1 & 27 & 1 & 0 & 0 & 0 & 28 & 1 & 0 & 0 \\
\hline CIN 2 & 35 & 0 & 7 & 26 & 2 & 0 & 0 & 3 & 32 & 0 & 0 \\
\hline CIN 3 & 63 & 0 & 0 & 8 & 55 & 0 & 0 & 0 & 3 & 60 & 0 \\
\hline SCC & 20 & 0 & 0 & 0 & 0 & 20 & 0 & 0 & 0 & 0 & 20 \\
\hline Total & 166 & 20 & 34 & 35 & 57 & 20 & 19 & 31 & 36 & 60 & 20 \\
\hline
\end{tabular}


compared with a "gold standard" made by the consensus of 3 gynaecopathology experts. When p16-immunostained slides were added as assistance, it was observed that the diagnostic accuracy for high-grade CIN was apparently increased. Sensitivity for high-grade CIN was increased by $13 \%$, and the false-negative rate decreased by half. Agreement of general pathologists in diagnosing high-grade CIN was significantly improved.

In this study, three experienced pathologists' "consensus diagnosis" with HE-stained slides was used as a standard. Consistency between the original HE diagnosis and the "consensus diagnosis", and consistency between the "diagnosis assisted by IHC" and the "consensus diagnosis" were compared. The consistency rate of the former was $88.55 \%$ while the consistency rate of the latter was $95.78 \%$. The latter was significantly higher than the former (Fisher's exact test, $\mathrm{p}=$ $=0.023$ ). It was found that diagnosis assisted by IHC had a higher diagnosis consistency rate than the original HE diagnosis. Our results show that the antibody cocktail of MCM2 and TOP2A, p16 and Ki-67 had sensitive and specific expressions in CIN and squamous cell carcinoma. They are reliable assistant indicators of CIN histological diagnosis and may increase the accuracy and reproducibility, and reduce inter-observer diagnostic variability. Immunohistochemical staining may be helpful in the interpretation of any individual cervical biopsy specimen, especially when a pathologist lacks sufficient experience.

\section{The authors declare no conflict of interest.}

\section{References}

1. Chen J, Chang XY. Diagnosis and differential of cervical intraepithelial neoplasias and related disease. Chin J Pathol 2007; 36: 563-566.

2. Mei P, Liu YH, Zhuang HG, et al. Diagnostic value of p16INK4a in squamous intraepithelial lesion in gynecologic cytology. Chin J Pathol 2007; 36: 521-523.

3. Galgano MT, Castle PE, Atkins KA, et al. Using biomarkers as objective standards in the diagnosis of cervical biopsies. Am J Surg Pathol 2010; 34: 1077-1087.

4. Pinto AP, Schlecht NF, Woo TY, et al. Biomarker (ProExTM C, p16INK4A, and MiB-1) distinction of high-grade squamous intraepithelial lesion from its mimics. Mod Pathol 2008; 21 : 1067-1074.

5. Regauer S, Reich O. CK17 and p16 expression patterns distinguish (atypical) immature squamous metaplasia from highgrade cervical intraepithelial neoplasia(CIN III). Histopathology 2007; 50: 629-635.

6. Bergeron C, Ordi J, Schmidt D, et al. Conjunctive p16INK4a testing significantly increases accuracy in diagnosing high-grade cervical intraepithelial neoplasia. Am J Clin Pathol 2010; 133: 395-406.

7. Cuschieri K, Wentzensen N. HPV mRNA and p16 detection as biomarkers for the improved diagnosis of cervical neoplasia. Cancer Epidemiol Biomarkers Prev 2008; 17: 2536-2545.

8. Han H, Yang Y, Lu Z, He Q, Lin Z, et al. Decreased D2-40 and increased p16INK4A immunoreactivities correlate with higher grade of cervical intraepithelial neoplasia. Diagn Pathol 2011; 6: 59 .
9. Lesnikova I, Lidang M, Hamilton-Dutoit S, Koch J. p16 as a diagnostic marker of cervical neoplasia: a tissue microarray study of 796 archival specimens. Diagn Pathol 2009; 4: 22.

10. Rosamilia C, Feichter G, Tzankov A, Obermann EC. Diagnosis and grading of cervical intraepithelial neoplasias. Pathologe 2012; 33: 118-123.

11. Martin CM, O'Leary JJ. Histology of cervical intraepithelial neoplasia and the role of biomarkers. Best Pract Res Clin Obstet Gynaecol 2011; 25: 605-615.

12. David O, Cabay RJ, Pasha S, et al. The role of deeper levels and ancillary studies (p16Ink4a and ProExC) in reducing the discordance rate of Papanicolaou findings of high-grade squamous intraepithelial lesion and follow-up cervical biopsies. Cancer 2009; 117: 157-166.

13. Shi J, Liu H, Wilkerson M, et al. Evaluation of p16INK4a, minichromosome maintenance protein 2, DNA topoisomerase IIalpha, ProEX C, and p16INK4a/ProEX C in cervical squamous intraepithelial lesions. Hum Pathol 2007; 38: 1335-1344.

14. Benevolo M, Mottolese M, Marandino F, et al. Immunohistochemical expression of p16INK 4a is predictive of HR-HPV infection in cervical low-grade lesions. Mod Pathol 2006; 19: 384391.

15. Branca M, Ciotti M, Santini D, el al. p16(INK4A) expression is related to grade of cin and high-risk human papillomavirus but does not predict virus clearance after conization or disease outcome. Int J Gynecol Pathol 2004; 23: 354-365.

16. Nam EJ, Kim JW, Hong JW, et al. Expression of the p16INK4a and Ki-67 in relation to the grade of cervical intraepithelial neoplasia and high-risk human papillomavirus infection. J Gynecol Oncol 2008; 19: 162-168.

17. Ma YY, Cheng XD, Zhou CY, et al. Value of P16 expression in the triage of liquid-based cervical cytology with atypical squamous cells of undetermined significance and low-grade squamous intraepithelial lesions. Chin Med J (Engl) 2011; 124: 2443-2447.

18. Tozawa-Ono A, Yoshida A, Yokomachi N, et al. Heat shock protein 27 and p16 immunohistochemistry in cervical intraepithelial neoplasia and squamous cell carcinoma. Hum Cell 2012; 25: 24-28.

19. Jackson JA, Kapur U, Eræahin Ç. Utility of p16, Ki-67, and HPV test in diagnosis of cervical intraepithelial neoplasia and atrophy in women older than 50 years with 3- to 7-year followup. Int J Surg Pathol 2012; 20: 146-153.

20. Kelly D, Kincaid E, Fansler Z, et al. Detection of cervical highgrade squamous intraepithelial lesions from cytologic samples using a novel immunocytochemical assay (ProEx C). Cancer 2006; 108: 494-500.

21. Ma Yuan-ying, Ye Feng, Chen Xiao-duan, et al. Evaluation of P16INK4a expression in Thinprep cervical specimens for the predication of high-grade cervical intraepithelial neoplasia. Natl Med J China 2010; 90: 3040-3044.

22. O'Neill CJ, McCluggage WG. p16 expression in the femal genital tract and its value in diagnosis. Adv Anat Pathol 2006; 13: $8-15$.

23. Lobato S, Tafuri A, Fernandes PÁ, et al. Minichromosome maintenance 7 protein is a reliable biological marker for human cervical progressive disease. J Gynecol Oncol 2012; 23: 11-15.

24. Walts AE, Bose S. P16/Ki-67 immunostaining is useful in stratification of atypical metaplastic epithelium of the cervix. Pathology 2008; 1: 35-42.

\section{Address for correspondence}

\section{Qi-chang Yang}

Department of Pathology

The $2^{\text {nd }}$ Affiliated Hospital of Nantong University

Hai Er Xiang North Road 6\#

226001 Nantong, China

e-mail: ntyangqc@163.com 